\section{Pandemic Prompted Pivoting to Virtual Multidisciplinary Care}

Brian D. Stephens, MD, MS', Hannah C. George, MS', Sara Ng ${ }^{1}$, Paul J. Sampognaro, $\mathrm{MD}^{1}$, Laura K. Rosow, $\mathrm{MD}^{1}$, Catherine Lomen-Hoerth, $\mathrm{MD}, \mathrm{PhD}^{1}$

${ }^{1}$ University of California, San Francisco; Department of Neurology; San Francisco, CA, USA

\section{Introduction}

The COVID-19 pandemic has drastically changed the way that neurologists deliver care to patients and has "catalyz[ed] the adoption of teleneurology". ${ }^{.}$Approximately $8 \%$ of hospitalized patients diagnosed with and treated for COVID-19 have a pre-existing neurological illness. ${ }^{2}$ Of these, patients with motor neuron disease and/or muscle disease, particularly those with bulbar or respiratory weakness, represent two of the most vulnerable groups. ${ }^{3}$

To provide continuity of care to these high risk individuals, the American Academy of Neurology has offered guidelines on implementing telemedicine for patients. ${ }^{4}$ However, in a recent survey of practicing neurologists, only $29 \%$ had access to a telehealth platform, and just $17 \%$ of practices had access to teleneurology in the outpatient setting. ${ }^{5}$ The pandemic has also led to the closure of most outpatient therapy centers, creating further difficulties in patient access to treatments. ${ }^{6}$

Despite these challenges, providing telehealth to patients with ALS has previously proven (at least on a smaller scale) to be feasible, provide high satisfaction, and maintain a quality of care similar to that of face-to-face visits. ${ }^{7,89}$

For these reasons, our MDA/ALS Clinic at the University of California, San Francisco (UCSF) have recently transitioned our in-person multidisciplinary clinics to a successful, virtual patient experience. Here, we outline our new clinic model, presenting detailed information about our clinics' virtual workflow and our experiences with this transition. In this way, we hope to demonstrate the feasibility of a large-scale virtual multidisciplinary clinic and assist other clinics (both local and academic) as they transition their care of patients virtually within the COVID-19 environment.

\section{About the UCSF ALS/MDA clinic}

Our multi-disciplinary team has members from 10 disciplines: respiratory therapy, physical therapy, occupational therapy, nutrition, speech language pathology, social work, research, patient care coordination, nursing, and neurology. Trainees from all disciplines often join our clinic as well. Prior to the COVID-19 pandemic, we served 20-25 patients every week, with 1-2 new patients and 3-6 follow ups every half day. As of May 26, 2020, we are serving approximately 15-20 patients every week solely through televisits. We expect this number to increase as we expand our practice beyond the California border in response to the easing of state medical licensure restrictions by the Centers for Medicare and Medicaid Services. ${ }^{10}$

\section{Software}

Our live video telehealth is conducted utilizing the Zoom Video Conferencing software with the waiting room feature enabled for enhanced security

\section{Prior to the Appointment Scheduling with Patients}

Our patient care coordinator discusses the nature of the telehealth appointment and ensures that patients have access to a device with a camera and microphone such as a smart phone, tablet, or computer. If the patient is using a smart phone or tablet, we ensure that they have the appropriate software application (in our case, the Zoom application) on the device and know how to operate it.

After completing the above process, our patient care coordinator sends the patient written instructions as a reminder on how to access the meeting on the day of their appointment.

\section{Scheduling with Providers}

Each member of our team has a unique meeting identification number (ID) that has been assigned to them through the Zoom interface. Our patient care coordinator chooses one of these numbers to provide to each individual patient for use during their appointment. By assigning individual numbers, we allow patients to remain in one virtual "room" for the duration of their visit. This approximates the experience of our regular clinic, in which patients remain in one room within clinic for the duration of their visit and eliminates issues with patients having to log-in to multiple rooms to different providers. Additional benefits to the virtual environment are that patients can share their Zoom link with family not living in the home and/or other health care providers involved in their care, so they can also join the visit if desired. 
To enable this workflow, all providers must grant access to the scheduler to allow them to create appointments using the meeting ID.

The scheduler then grants Host access as an "Alternate Host" to all members of our team. This allows any given team member to start the meeting independently and must be done for every patient individually.

\section{Assessments to Assist with the Visit}

One day prior to the appointment, a member of our team will call the patient to conduct functional rating scales (such as the ALS-FRS-R and CNS-LS), perform medication reconciliation, and discuss specific issues the patient would like to bring up with our team. These are documented in the medical record.

\section{Day of the Appointment Clinical Flow}

We establish one Team Meeting Room (with a unique Zoom ID) where all members of the multi-disciplinary team can discuss patient-specific issues at the start of the day. An online Google Sheets spreadsheet is accessible by team members and lists the patients' initials (full names are not used to remain HIPAA-compliant), the Zoom IDs being used for their visit, and notes about their care. Team members (neurology, PT, etc.) are listed in the columns of the spreadsheet, and individual providers can mark their time in and out of the patient's room (Figure 1). This spreadsheet can be screen-shared via Zoom and directly annotated through the program if operating numerous screens proves challenging. A coordinator is usually present throughout clinic and helps to manage provider workflow and ensure that the spreadsheet is being updated correctly.

\section{Patient Visit}

Approximately 15-30 minutes prior to the visit, our patient care coordinator will call the patient to guide them through the process of joining the virtual room and troubleshooting issues, as necessary.

\section{Waiting Rooms}

One option is utilizing the Waiting Room feature in Zoom to ensure confidentiality. One of the team members joins the meeting first (as the Host) and will allow the appropriate patient into the room from the Waiting Room. At the conclusion of this visit, we then make the patient a "co-host," so they can remain in the room and "admit" the next provider when they arrive. If the patient's condition restricts their ability to admit the next provider, we either ensure there is a caregiver with them, or a team member will remain in the room until the next provider joins.

\section{Breakout Rooms}

As an alternative to using multiple separate meeting IDs, Zoom now has the option of "breakout rooms," which allow multiple separate meeting spaces under one provider's Zoom ID. A coordinator still admits patients to the meeting, then "rooms" patients and caregivers/family members in separate breakout rooms, identified by patient name. Providers still congregate and discuss patients in a Team Meeting Room, then move directly into the separate patient visit rooms. Extra meeting rooms are usually created as well, which can be useful for smaller team discussions (e.g., an attending physician staffing a patient with a trainee). Patients are unable to view the different breakout rooms, thereby maintaining HIPAA compliance.

The use of breakout rooms has several potential benefits over the use of separate Zoom IDs. For one, the list of available breakout rooms includes a list of current participants in each space, thereby allowing providers to view who is in a room with a patient at any given time. Breakout rooms also allow the use of one consistent Zoom ID (e.g., that of the attending physician) over time, decreasing the likelihood of patients or providers having the wrong meeting ID. Patients and caregivers do not need to "admit" providers to their room, which can be technically challenging for some. Additionally, breakout rooms eliminate the need to log in and out of multiple meetings over the course of a single day, instead allowing providers to remain logged into

Figure 1: Example of a Virtual Clinic Spreadsheet

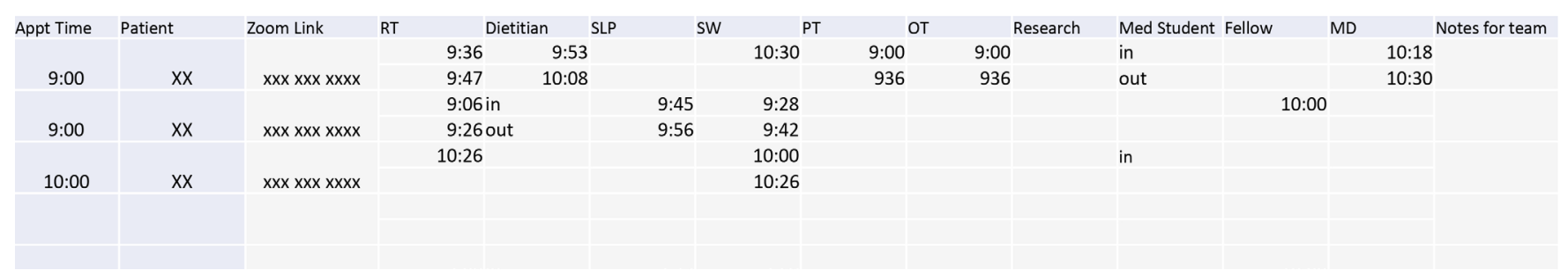


Figure 2: A Typical Clinic Day for the Patient and Team Members

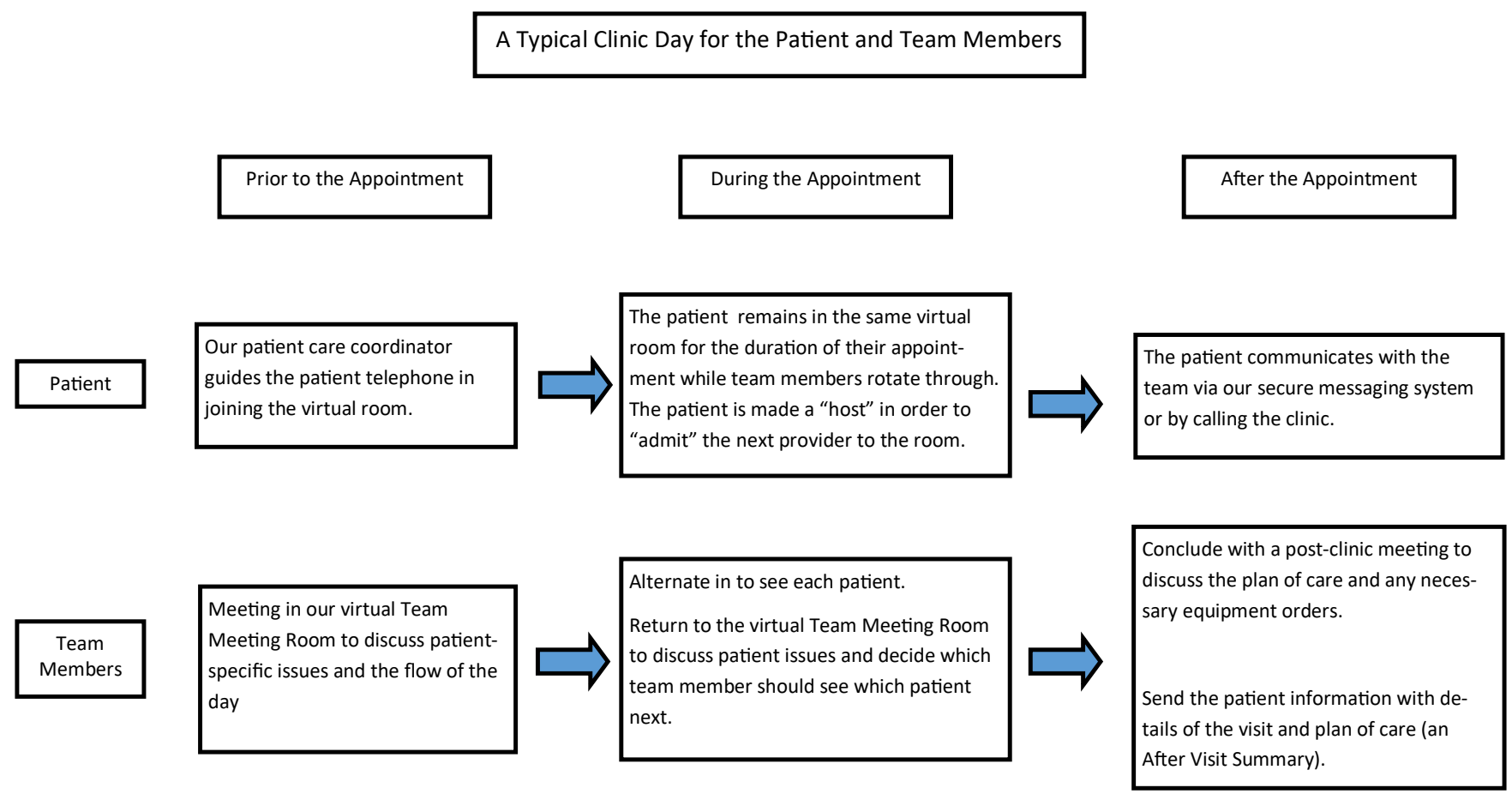

one Zoom meeting for the duration of the clinic. We have recently begun using breakout rooms instead of separate meeting IDs, and providers seem to prefer this option for the many reasons listed above.

If a learner (medical student, resident, etc.) is joining us, we typically have the learner remain in the room for the majority of the visit, both for patient continuity and to manage the flow of providers entering and exiting the room.

\section{After the Appointment}

We end with a post-clinic meeting in the virtual Team Meeting Room to discuss the plan of care and any necessary equipment orders. Patients are encouraged to communicate with us via our secure messaging system (Epic's MyChart function) or by calling our clinic. Patient instructions can be entered electronically and are automatically messaged to the patient as the visit is finalized. If the patient does not have electronic access, these instructions can be printed and mailed.

\section{Specific Care Considerations}

\section{Respiratory Care}

A drawback for virtual respiratory care is that we are unable to perform pulmonary function testing with formal spirometry on those with an unknown COVID-19 status.
Spirometry is an aerosolized procedure and likely will continue to be restricted as long as COVID-19 remains a significant concern even after in-person patient visits are allowed. This testing - and its trajectory over time -- typically guides recommendations regarding respiratory equipment and discussions of prognosis. While some outpatient or hospital-based laboratories have recently restarted spirometry testing patients with proof of COVID-19 negativity, in an effort to help detect neuromuscular respiratory weakness in the absence of spirometry testing, we are ordering more nocturnal oximetry in symptomatic patients to assess for nocturnal hypoventilation. Symptoms of shortness of breath or an abnormal nocturnal oximetry study now qualify patients for noninvasive positive-pressure ventilation (NPPV) due to relaxation of insurance requirements.

\section{Physical and Occupational Therapy}

While our therapists are no longer able to evaluate motor function in-person, we are now able to see patients in their home environment, where we can more readily evaluate for safety. Fortunately, due to recent insurance policy changes, we are also now able to order wheelchairs and other durable medical equipment (DME) for our patients without an inperson visit being required. 


\section{Palliative Care}

Our palliative care specialists have been early adopters of telehealth, as this specialty lends itself particularly well to remote evaluation and counseling of patients. ${ }^{11}$ Benefits of telehealth palliative care include the ability to support patients close to the end of life in a more comfortable setting, and the ability of family members/caregivers to join the visit from multiple different locations.

\section{Clinical Trials}

Clinical trials have largely been put on hold, so no new patients are being recruited at this time. For patients that were already enrolled, telehealth is utilized, and urgent/medically necessary issues are addressed in person.

\section{Conclusion}

While the COVID-19 Pandemic has changed the way we all care for our patients, comprehensive, multidisciplinary care is still possible through telehealth visits. We have continually worked to refine our process over the last three months. While every clinic must find a method that will address the challenges of its own system, we hope this outline serves as a framework for the successful adoption of telehealth multidisciplinary care.

\section{Corresponding author:}

Brian Stephens

stephensbd@gmail.com

\section{References}

${ }^{1}$ Klein BC, Busis NA. COVID-19 is catalyzing the adoption of teleneurology. Neurology 2020;94:903-904. doi:10.1212/WNL.0000000000009494

2 Herman C, Mayer K, Sarwal A. Scoping review of prevalence of neurologic comorbidities in patients hospitalized for COVID-19. Neurology Apr 2020, 10.1212/WNL.0000000000009673; DOI: 10.1212/ WNL.0000000000009673

${ }^{3}$ Association of British Neurologists. Association of British Neurologists guidance on COVID-19 for people with neurological conditions, their doctors and carers. Published March 22, 2020. Available at: https://cdn.ymaws. com/www.theabn.org/resource/ collection/6750BAE64CBC-4DDB-A684-116E03BFE634/ABN_Neurology_ COVID-19_Guidance_22.3.20.pdf. Accessed on May 26, 2020 .
${ }^{4}$ Telemedicine and COVID-19 Implementation Guide. American Academy of Neurology. Updated April 10, 2020. Accessed 26May2020.

${ }^{5}$ Sharma A, Maxwell CR, Farmer J, Greene-Chandos D, LaFaver K, Benameur K. Initial experiences of US neurologists in practice during the COVID-19 pandemic via survey. Epub ahead of print 21May2020. NEUROLOGY DOI: 10.1212/WNL.0000000000009844

${ }^{6}$ Lin C. Invited Commentary: Advancing telerehabilitation in the time of COVID-19. Neurology Blogs. 11May2020. https://blogs.neurology.org/covid-19-coronavirus/invitedcommentary-advancing-telerehabilitation-in-the-time-ofcovid-19/. Accessed 26May2020

${ }^{7}$ Van De Rijn M, Paganoni S, Levine-Weinberg M, et al. Experience with telemedicine in a multi-disciplinary ALS clinic. Amyotroph Lateral Scler Frontotemporal Degener. 2018;19:143-148. https://doi.org/10.1080/21678421.2017.1 392577.

${ }^{8}$ Geronimo A, Wright C, Morris A, Walsh S, Snyder B, Simmons Z. Incorporation of telehealth into a multidisciplinary ALS Clinic: feasibility and acceptability. Amyotroph Lateral Scler Frontotemporal Degener. 2017;18:555-561. https://doi.org/10.1080/21678421.2017.1338298.

${ }^{9}$ Selkirk SM, Washington MO, McClellan F, Flynn B, Seton JM, Strozewski R. Delivering tertiary centre specialty care to ALS patients via telemedicine: a retrospective cohort analysis, Amyotrophic Lateral Sclerosis and Frontotemporal Degeneration, 2017, 18:5-6, 324-332, DOI: 10.1080/21678421.2017.1313867

${ }^{10}$ Center for Connected Health Policy. Telehealth Coverage Policies in the Time of COVID-19. Available at: cchpca.org/resources/covid-19-telehealth-coverage-policies. Accessed May 27, 2020.

11 Jess M, Timm H, Dieperink KB. Video Consultations in Palliative Care: A Systematic Integrative Review. Palliat Med. 2019 Sep;33(8):942-958. doi: $10.1177 / 0269216319854938$. 